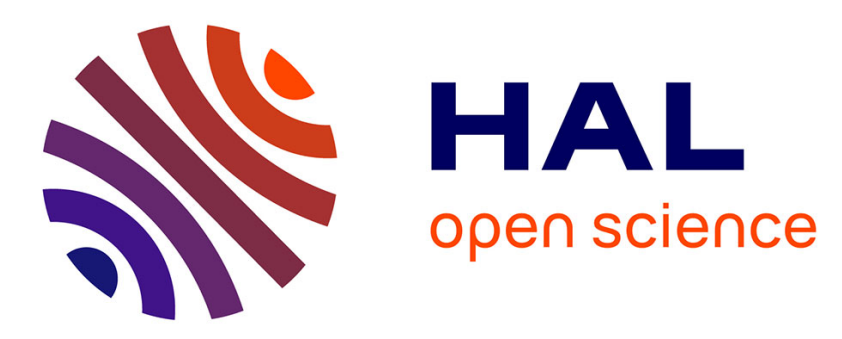

\title{
Thermal conductivity of deca-nanometric patterned Si membranes by multiscale simulations
}

\author{
Hayat Zaoui, Pier Luca Palla, Stefano Giordano, Fabrizio Cleri, Maxime \\ Verdier, David Lacroix, J.F. Robillard, Konstantinos Termentzidis, Évelyne \\ Martin
}

\section{To cite this version:}

Hayat Zaoui, Pier Luca Palla, Stefano Giordano, Fabrizio Cleri, Maxime Verdier, et al.. Thermal conductivity of deca-nanometric patterned $\mathrm{Si}$ membranes by multiscale simulations. International Journal of Heat and Mass Transfer, 2018, 126, pp.830-835. 10.1016/j.ijheatmasstransfer.2018.06.004 . hal-01826181

\section{HAL Id: hal-01826181 \\ https://hal.science/hal-01826181}

Submitted on 29 Jun 2018

HAL is a multi-disciplinary open access archive for the deposit and dissemination of scientific research documents, whether they are published or not. The documents may come from teaching and research institutions in France or abroad, or from public or private research centers.
L'archive ouverte pluridisciplinaire HAL, est destinée au dépôt et à la diffusion de documents scientifiques de niveau recherche, publiés ou non, émanant des établissements d'enseignement et de recherche français ou étrangers, des laboratoires publics ou privés. 


\title{
Thermal conductivity of deca-nanometric patterned Si membranes by multiscale simulations
}

\author{
Hayat Zaoui ${ }^{\mathrm{a}}$, Pier Luca Palla ${ }^{\mathrm{a}}$, Stefano Giordano ${ }^{\mathrm{a}}$, Fabrizio Cleri ${ }^{\mathrm{a}}$, Maxime Verdier ${ }^{\mathrm{b}}$, David Lacroix $^{\mathrm{b}}$, \\ Jean-François Robillard ${ }^{a}$, Konstantinos Termentzidis ${ }^{\text {b,c }}$, Evelyne Martin ${ }^{\mathrm{a}, *}$ \\ ${ }^{\text {a } U n i v . ~ L i l l e, ~ C N R S, ~ C e n t r a l e ~ L i l l e, ~ I S E N, ~ U n i v . ~ V a l e n c i e n n e s, ~ U M R ~} 8520$ - IEMN, F-59000 Lille, France \\ ${ }^{\mathrm{b}}$ Université de Lorraine, CNRS, LEMTA-UMR7563, Vandœuvre-lès-Nancy F-54500, France \\ ${ }^{\mathrm{C}}$ Université de Lyon, CNRS, INSA-Lyon, Université Claude Bernard Lyon 1, CETHIL UMR 5008, F-69621 Villeurbanne, France
}

\section{A R T I C L E I N F O}

\section{Article history:}

Received 17 January 2018

Received in revised form 19 April 2018

Accepted 1 June 2018

\section{Keywords:}

Thermal conductivity

Silicon membranes

Simulation

\begin{abstract}
A B S T R A C T
The hollowing of silicon membranes to form a lattice of cylindrical holes, also called phononic crystal, has been used by several experimental groups willing to fabricate efficient thermoelectric modules. The idea is to reduce the thermal conductivity without impacting the electronic conductivity. For several a priori identical materials, i.e. thin films containing periodic cylindrical holes, drastically different levels of thermal conductivity reduction have been reported in the literature: from $1-2 \mathrm{~W} \mathrm{~K}^{-1} \mathrm{~m}^{-1}$ to $15-40 \mathrm{~W} \mathrm{~K}^{-1}$ $\mathrm{m}^{-1}$, i. e. half the thermal conductivity of the plain membrane. These differences may be due to variations in the geometrical patterns, or to the technological processes specific to each group. It is therefore highly desirable to understand which level of reduction can be expected from the basic concept. In this work, we address the question by applying a fully atomistic framework, the approach-to-equilibrium molecular dynamics (AEMD), to study two deca-nanometric patterns used in the literature and reported respectively with a high and low level of thermal conductivity reduction. For both patterns, the thermal conductivity roughly decreases by a factor 2 only compared to the plain membrane. Thanks to Monte Carlo simulations, in agreement with AEMD for the two patterns, we propose that the origin of stronger reductions could be an increase of the surface roughness during the step of hole fabrication.
\end{abstract}

\section{Introduction}

Nanostructuration opens perspectives in the field of thermoelectricity to materials that are not a priori good candidates. This is the case for crystalline silicon, a safe and abundant material whose technology has been largely developed and that one would like to use on the same chip to process the information and handle the energy conversion. Several options of nanostructuration have been studied, such as the 1D structuration in nanowires combined with an optimisation of the surface roughness [1-3]. In the same idea of limiting the phonon free paths to decrease the thermal conductivity, the fabrication of nanostructured membranes including nano-holes also raised a great interest [4-8]. In silicon at room temperature, $80 \%$ of the total thermal conductivity is provided by phonons that experience mean free paths larger than $100 \mathrm{~nm}$ $[9,10]$. The pattern dimensions (hole diameter and pitch) should ideally be choosen in the range $10-100 \mathrm{~nm}$ to act as efficient cutoffs on the phonon MFP distribution and strongly reduce the

\footnotetext{
* Corresponding author.

E-mail address: evelyne.lampin@univ-lille.fr (E. Martin).
}

thermal conductivity with a limited impact on the electronic conductivity (MFPs of a few nanometers). Patterns in this range are however challenging to obtain even by the most advanced nanofabrication techniques, but a cutoff at $500 \mathrm{~nm}$ can still suppress half the total thermal conductivity [9].

However, there is a controversy on the level of thermal conductivity reduction resulting from nanostructuration. In Refs. [4,5], values of $1-2 \mathrm{~W} \mathrm{~K}^{-1} \mathrm{~m}^{-1}$, close to the amorphous limit are reported, while in Refs. [6-8], the thermal conductivity only reduces to $15-40 \mathrm{~W} \mathrm{~K}^{-1} \mathrm{~m}^{-1}$, i. e. half the thermal conductivity of the plain membrane. The discrepancy can not be attributed to the range of dimensions and volume fraction, since it is similar in Refs. [4,5,7] (between 10 and $100 \mathrm{~nm}$ and 10-30\%) and in Refs. $[4,6,8]$ (several hundreds of $\mathrm{nm}$ and $\approx 35 \%$ ). It is therefore highly desirable to be able to distinguish between the effect of nanostructuration and of specific nano-fabrication methods by simulating holey silicon membranes free of any defects but with real dimensions.

In the present work, we focus on two membranes falling in the deca-nanometric range: the membranes of Refs. [5] (hereafter called "Y") and [7] (hereafter called " $H$ "). The characteristic lengths 
of the plain and nanostructured membranes are defined in the schematical representation of Fig. 1. Their values are reported in Table 1. The thermal conductivities measured in Ref. [5] are equal to $17 \mathrm{~W} \mathrm{~K}^{-1} \mathrm{~m}^{-1}$ for the plain membrane and $1-2 \mathrm{~W} \mathrm{~K}^{-1} \mathrm{~m}^{-1}$ for the nanostructured membrane, while in Ref. [7], the thermal conductivity are equal to $59 \pm 10 \mathrm{~W} \mathrm{~K}^{-1} \mathrm{~m}^{-1}$ for the plain membrane and $34.5 \pm 7.5 \mathrm{~W} \mathrm{~K}^{-1} \mathrm{~m}^{-1}$ for the nanostructured membrane. The higher absolute values obtained in Ref. [7] can be explained by the greater thickness of their membranes. However, the reduction of thermal conductivity is far more pronounced in Ref. [5] $(\approx 10)$ than in Ref. [7] $(<2)$.

The thermal conductivity of nanostructured membranes (hereafter called "L") has been studied by molecular dynamics (MD) simulations [11] using the Green-Kubo approach [12]. The patterns $\mathrm{Y}, \mathrm{H}$ and $\mathrm{L}$ are compared in Fig. 2. A thermal conductivity of $45 \mathrm{~W}$ $\mathrm{K}^{-1} \mathrm{~m}^{-1}$ has been obtained for the plain membrane, that decreases to 2 to $12 \mathrm{~W} \mathrm{~K}^{-1} \mathrm{~m}^{-1}$ for hole diameters ranging from $D=3-6.5$ $\mathrm{nm}$. The thickness and pitch were set to $t=8.1 \mathrm{~nm}$ and $p=8.1$ $\mathrm{nm}$. The reduction of thermal conductivity is significative, and ranges from 4 to 25 . However the dimensions are considerably lower dimensions than in experiments, and it can not be ruled out that the thermal conductivity strong reduction is due a stronger downscaling.

In order to achieve a better understanding of the thermal conductivity of nanostructured membranes, we have studied the three patterns by using three complementary theroretical approaches, going from the atomistic level (molecular dynamics), to the mesoscopic level (Monte Carlo) and to the continuum level (effective medium theory). This work is presented as follows. Section 2 provides details on the MD method and presents the results. Section 3 is devoted to the other modelling work by Monte Carlo simulations and using the effective medium theory. Section 4 contains the comparison with experiments and discussion of our results. Concluding remarks are collected in Section 5.

\section{Approach-to-equilibrium molecular dynamics}

We have started by studying a plain membrane (hereafter called "P") to obtain a reference of thermal conductivity in absence of nanostructuration. The plain membrane is modeled by an elementary rectangular pattern presented in Fig. 1 (dasheddelimited volume). The atoms are arranged on a diamond lattice and the upper and lower surfaces are smooth. Periodic boundary conditions are applied in the two directions of the membrane
Table 1

Thickness $t$, hole diameter $D$ and pitch $p$ of patterns.

\begin{tabular}{cccc}
\hline Source & $t(\mathrm{~nm})$ & $D(\mathrm{~nm})$ & $p(\mathrm{~nm})$ \\
\hline Ref. [5] ("Y") & 22 & 16 & 34 \\
Ref. [7] ("H") & 54 & 20 & 60 \\
Ref. [11] ("L") & 8.1 & 5.4 & 8.1 \\
\hline
\end{tabular}

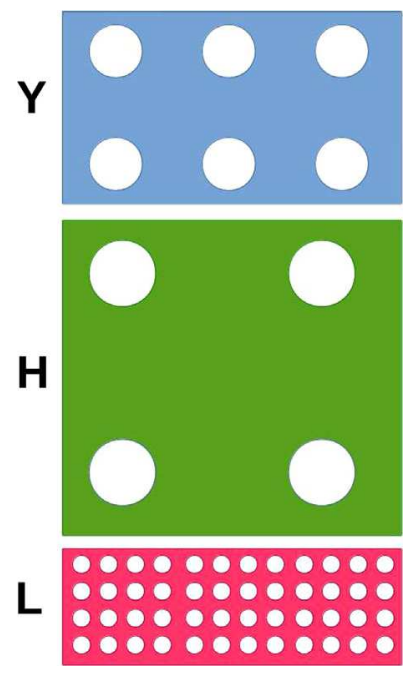

Fig. 2. $Y, H$ and $L$ patterns drawn on the same scale.

plane. In the present study, the thickness has been fixed to $t=8.1 \mathrm{~nm}$ (15 lattice units) for all the membranes. The square section of the rectangular supercell that models the plain membrane has a side equal to $a=t=8.1 \mathrm{~nm}$. It has been shown [19] that the dependence of the thermal conductivity on the cross section of the simulation cell is weak with the MD methodology presented below. On the other hand, the thermal conductivity exhibits a pronounced dependence on length, both in bulk materials [19] and nanowires [20], and the length $L$ must therefore be increased until the thermal conductivity becomes constant in order to guarantee that the thermal conductivity does not present any size effects inherent to the method, and can be compared to other theoretical or experimental values. In the present case and for the plain membranes, this has required to increase the length $L$ from 80 up to $800 \mathrm{~nm}$.

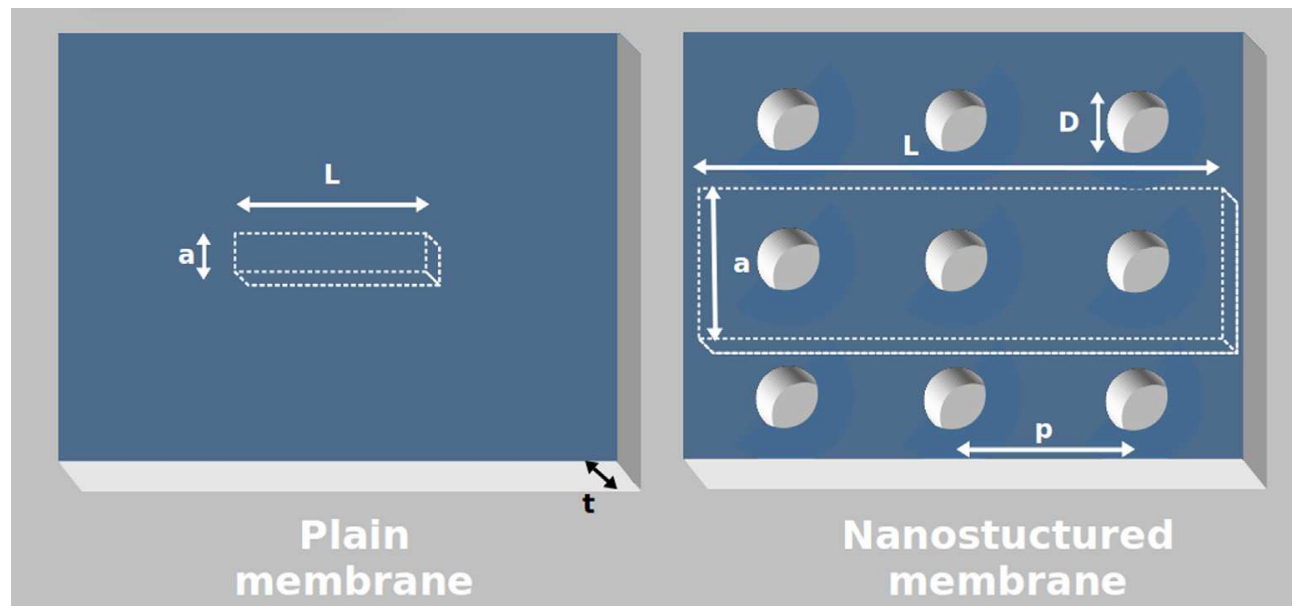

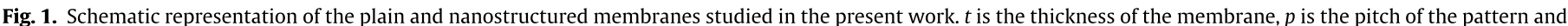

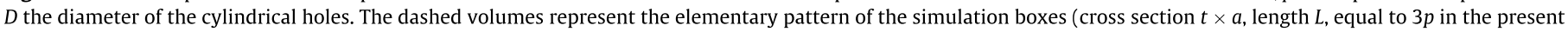
case). 
The elementary pattern build to model the nanostructured membrane is a rectangular box of thickness $t=8.1 \mathrm{~nm}$, and sides $a=L=p$ (dashed-delimited volume in Fig. 1, right). In order to get rid of any length dependence, the elementary supercell $p \times p$ is duplicated one time $p \times(L=2 p)$, 2 times $p \times(L=3 p)$, . . until the thermal conductivity becomes constant. In the case of the pattern Y, the elementary supercell contains $N_{a t}=380,000$ atoms and has been replicated up to 8 times ( $N_{a t} \approx 3$ millions of atoms). In the case of the pattern $\mathrm{H}$, the elementary supercell contains $N_{a t}=1.3 \mathrm{mil}-$ lions of atoms and has been replicated up to 5 times $\left(N_{a t} \approx 6.4\right.$ millions of atoms). We have also studied the smaller structure (pattern L), having in mind the comparison of our results with those from a different MD methodology.

The MD simulations are carried out using a modified version of the code DL_POLY [14]. The interactions between atoms are modeled by Tersoff's empirical potential [13]. After equilibration of the structure at room temperature $T_{t}$, the approach-to-equilibrium molecular dynamics (AEMD) methodology $[15,16]$ is applied. In phase 1 , the initial state is created by applying simultaneously two Nosé-Hoover $[17,18]$ thermostats, a first one at a hot temperature $(400 \mathrm{~K})$ and a second thermostat at a cold temperature (200 $\mathrm{K})$ respectively to the first and second halves of the atomic box along the length $L$. Fig. 3.a shows the average temperature in each block, $T_{1}$ and $T_{2}$, that are equilibrated during $30 \mathrm{ps}$. In Fig. $3 \mathrm{~b}$, the temperature profiles averaged over the last 10 ps of the trajectory are presented. Parabola are superimposed at the minimum and maximum, a sign that the temperature difference is correctly equilibrated (the heat equation including a generation term $G$ for the thermostat is reduced in the steady state to $\frac{\partial^{2} T}{\partial z^{2}}=-G / D=$ constant, with $D$ the thermal diffusivity). For the nanostructured membrane, the supercell contains two holes (i.e. the elementary supercell is replicated once), centered are at the minimum and at the maximum of the temperature profiles. This is the reason why the profile presents a bending at the neck (around $z / L=0$ and $z / L= \pm 0.5$ ). In phase 2 of the AEMD, the thermostats are removed and the equilibrium is approached during a microcanonical trajectory. In Fig. 3.c, the temperature difference $\Delta T=T_{2}-T_{1}$ between the average temperatures of the hot and cold blocks is plotted for the plain and the nanostructured membrane. The decay is exponential, as evidenced by the curves superimposed on the MD profiles. In the case of the nanostructured membrane, a short transient first occurs, and a bi-exponential curve adjusts more properly the profile. The larger of the two decays, that corresponds to the asymptotic behaviour, is exploited to obtain the thermal conductivity, according to the procedure explained below. In Fig. 3.d, the temperatures profiles in phase 2 are plotted. In dark, the profile in the plain membrane follows a sine function of the form $\sin (2 \pi z / L)$, with a small deviation at the minimum and maximum. In red, the temperature profile in the nanostructured membrane is also a sine function in average, but with slight curvature variations between the regions with and without hole.

The results of the MD simulations are analysed at the light of the heat equation, written and solved in the same conditions. The procedure has already been applied in the case of bulks $[16,19]$ and nanowires [20]. The temperature difference between the two blocks has a dominant and asymptotic contribution of the form:

$\Delta T(t) \propto \Delta T_{0} e^{-t / \tau_{D}}$

and the temperature profile $T(z)$ is a Fourier series with a dominant sinusoidal term of decaying amplitude $\sin (2 \pi z / L) e^{-t / \tau_{D}}$. Since both the temporal evolution of the temperature difference and the temperature profiles in phase 2 (Fig. 3. c and.d) are consistent with the transient solution of the heat equation, we can exploit the relation a) Phase 1, T(t)

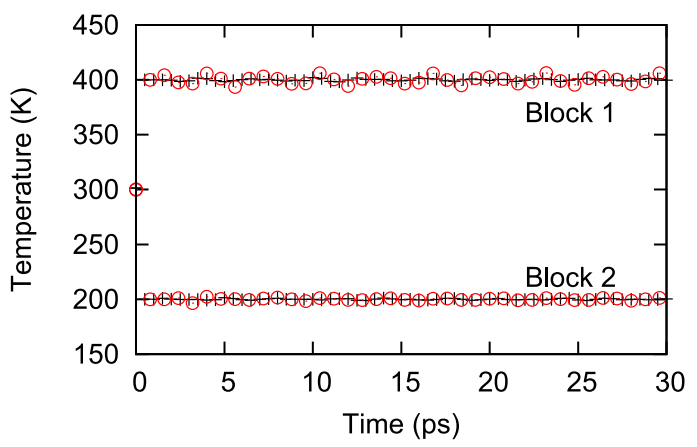

c) Phase 2, $\Delta \mathrm{T}(\mathrm{t})$

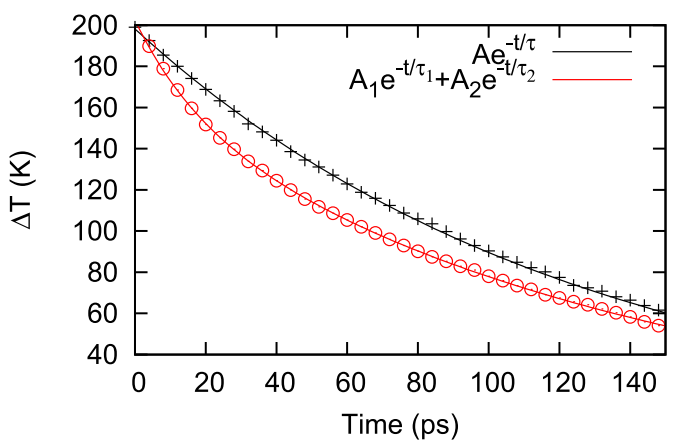

b) Phase 1, T(z)

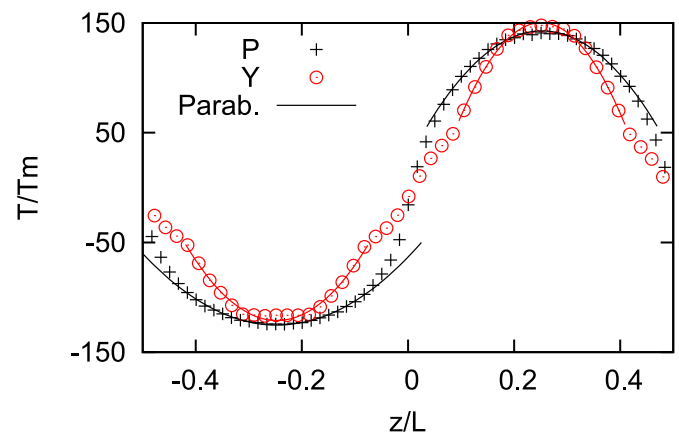

d) Phase 2, $T(z)$

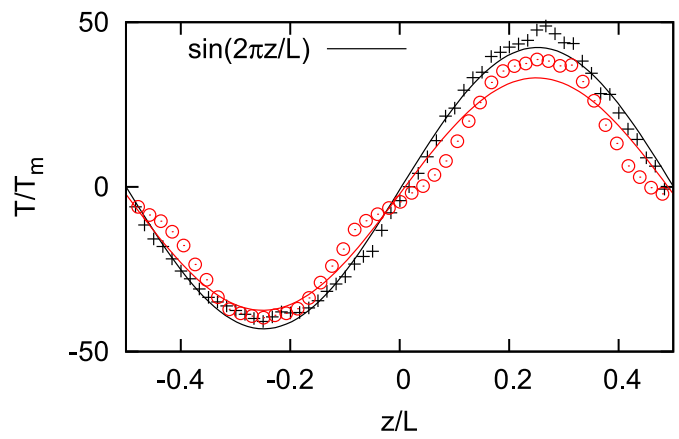

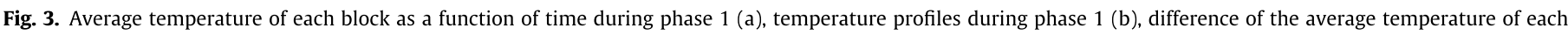

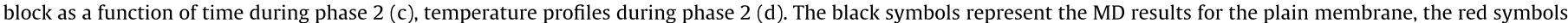

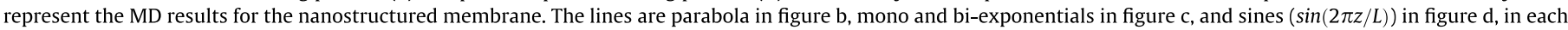
case adjusted to the MD results. (For interpretation of the references to colour in this figure legend, the reader is referred to the web version of this article.) 
between the decay time $\tau_{D}$ (provided the asymptotic decay is used) and the thermal conductivity derived from the heat equation following the relation:

$\kappa=\frac{L^{2}}{4 \pi^{2}} \frac{C_{V} \cdot \rho}{\tau_{D}}$

where $\rho=N_{a t} / V$ is the number density and $C_{V}=3 k_{\mathrm{B}}$ the specific heat. The volume $V$ is equal to $t^{2} L$ in the case of the plain membrane, and to

$V=t\left(a L-n_{\mathrm{h}} \frac{\pi D^{2}}{4}\right)$

for a nanostructured membrane containing $n_{\mathrm{h}}$ holes. The decay time being extracted from the difference of average temperatures in each block, the thermal conductivity obtained from Eq. (2) is an average value that does not account for the details of the temperature profile, and corresponds to the macroscopic value as determined by the experimental measurements.

The thermal conductivities obtained by the AEMD methodology are presented in Fig. 4. For all the membranes studied in the present work, we are able to observe the thermal conductivity become constant at large $L$. For the plain membrane P, the conductivity saturates to a value of $\kappa_{\mathrm{MD}}^{\mathrm{P}}=45 \pm 5 \mathrm{~W} \mathrm{~K}^{-1} \mathrm{~m}^{-1}$. This value is larger than the experimental value $\left(9 \mathrm{~W} \mathrm{~K}^{-1} \mathrm{~m}^{-1}\right.$ for a $9 \mathrm{~nm}$-thick membrane [21]). It has been shown [22] that this overestimation occurs because the surface of the MD-simulated membrane has no roughness or oxide passivation, as opposed to the nanofabricated structures. In the present work, we focus on the impact on the thermal conductivity of the nanostructuration by hole hollowing. Therefore, we will consider the factor of reduction from a plain to a nanostructured membrane, and not the absolute values of thermal conductivities. A last point concerning the plain membrane is the length $L^{\mathrm{P}} \approx 600 \mathrm{~nm}$ that corresponds to the thermal conductivity saturation, identifying the value of the maximum phonon free paths in the structure [19]. The thermal conductivity saturates to $\kappa_{\mathrm{MD}}^{\mathrm{H}}=21.2 \pm 0.1 \mathrm{~W} \mathrm{~K}^{-1} \mathrm{~m}^{-1}$ above lengths $L^{\mathrm{H}}=200 \pm 50 \mathrm{~nm}$ for the pattern of Ref. [7], to $\kappa_{\mathrm{MD}}^{\mathrm{Y}}=17.2 \pm 0.1 \mathrm{~W} \mathrm{~K} \mathrm{~K}^{-1} \mathrm{~m}^{-1}$ above lengths $L^{\mathrm{Y}}=200 \pm 10 \mathrm{~nm}$ for the pattern of Ref. [5], and to $\kappa_{\mathrm{MD}}^{\mathrm{L}}=3.4 \pm 0.2 \mathrm{~W} \mathrm{~K}^{-1} \quad \mathrm{~m}^{-1}$ above lengths $L^{\mathrm{L}}=80 \pm 10 \mathrm{~nm}$ for

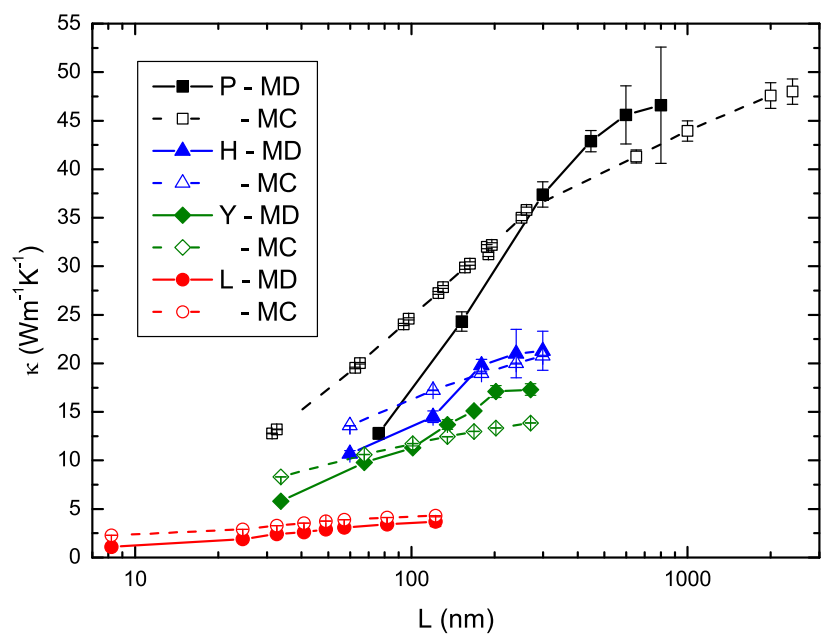

Fig. 4. Thermal conductivity versus length obtained in the plain and nanostruc tured membranes by MD (filled symbols) and MC (open symbols). The results for the plain membranes $\mathrm{p}$ are represented by squares, for the nanostructured membranes $\mathrm{H}$ by triangles, for the nanostructured membranes $\mathrm{Y}$ by diamonds and for the nanostructured membranes $L$ by circles. The lines connecting the points are guides for the eyes. the pattern of Ref. [11]. This last thermal conductivity is in good agreement with the theoretical result of Ref. [11] obtained with the interatomic potential of Ref. [28].

\section{Monte Carlo simulation and effective medium theory}

The same membranes have also been studied by Monte Carlo simulations. The latter technique is based on the resolution of the Boltzmann Transport Equation (BTE) for phonons in the relaxation time approximation [23-25]:

$\frac{\partial f}{\partial t}+\nabla_{\mathbf{K}} \omega \cdot \nabla_{\mathbf{r}} f=\frac{f^{0}-f}{\tau_{R}(\omega, T)}$

$f$ is the phonon distribution function at temperature $T, v_{g}=\nabla_{\mathbf{K}} \omega$ is the group velocity, $\tau_{R}$ is the relaxation time, and the exponent " 0 " refers to thermal equilibrium. In this approach, phonons are considered as pseudoparticles with wave properties (pulsation, group velocity and polarization) carrying a given energy $\hbar \omega$ and interacting through phonon-phonon processes, Rayleigh scattering with impurities and boundary scattering with the geometrical boundaries of the nanostructure. The BTE (Eq. (4)) is solved thanks to an iterative algorithm where phonon displacements and interactions are modeled through several steps: (i) Design of the geometry of the structure (plain or nanostructured) and discretization of the control volumes. The MC control volumes are the same as supercells used in MD simulations presented above. (ii) Boundary condition setting. First and last cells are set to prescribed temperatures (blackbodies), phonon reflection at hole edges and lateral boundaries ( $y$ direction) is specular while it depends on the specularity parameter $p$ on the top and bottom edges of the membrane ( $x$ direction). (iii) Sampling of phonon population (frequency, polarization and group velocity, using an energy cumulative distribution function [24]) at random space positions in a cell according to its prescribed temperature. Phonon properties are those of the bulk crystalline Si using data provided by Pop et al. [27]. (iv) Advective transport of phonons during a time step $\delta t$ according to their initial position and group velocity. Reflections at the boundaries (membrane and hole edges) are achieved during this step. v) Phonon internal scattering. The scattering probability depends on phonon relaxation time as $P_{\text {scat }}=1-\exp \left(-\delta t / \tau_{R}\right)$, the selection of Umklapp, normal or impurity scattering is done through random number sampling. Phonon properties (frequency, polarization, group velocity and propagation direction) are partly or fully resampled depending on the considered process. Semiempirical lifetimes proposed by Holland [26] are used in this work.

During MC simulation, the three last steps are repeated until steady state is reached (after tens of nanoseconds depending on the nanostructure length). The heat flux along $z$ is given as the energy transported by phonons in each cell $\mathbf{J}_{z}=\frac{1}{V} \sum \hbar \omega \mathbf{v}_{\mathrm{gz}}$ of volume $V$. Both heat flux and temperature are averaged on the steady state period, than the thermal conductivity is extracted according to the Fourier's law as $\mathbf{J}_{z}=-\kappa \nabla T$. More details about the simulation procedure can be found in references [23-25].

The results of the MC calculations are also plotted in Fig. 4. The length dependences are similar using the two approaches. The thermal conductivites converge to a value of $\kappa_{\mathrm{MC}}^{\mathrm{P}}=47.0 \pm 1.5 \mathrm{~W}$ $\mathrm{K}^{-1} \mathrm{~m}^{-1}$ for the plain membrane, $\kappa_{\mathrm{MC}}^{\mathrm{H}}=24.2 \pm 0.2 \mathrm{~W} \mathrm{~K}^{-1} \mathrm{~m}^{-1}$ for the nanostructured membrane $\mathrm{H}, \kappa_{\mathrm{MC}}^{\mathrm{Y}}=15.7 \pm 0.1 \mathrm{~W} \mathrm{~K}^{-1} \mathrm{~m}^{-1}$ for the nanostructured membrane $\mathrm{Y}$ and $\kappa_{\mathrm{MC}}^{\mathrm{L}}=5.2 \pm 0.0 \mathrm{~W} \mathrm{~K}^{-1} \mathrm{~m}^{-1}$ for the nanostructured membrane $L$. The results of the two approaches, MD and MC, are in good agreement. It can therefore be concluded that the downscaling does not impact significantly the phononic states (since the bulk characteristics are used in the MC calculations) and that a classical treatment is proper (since 


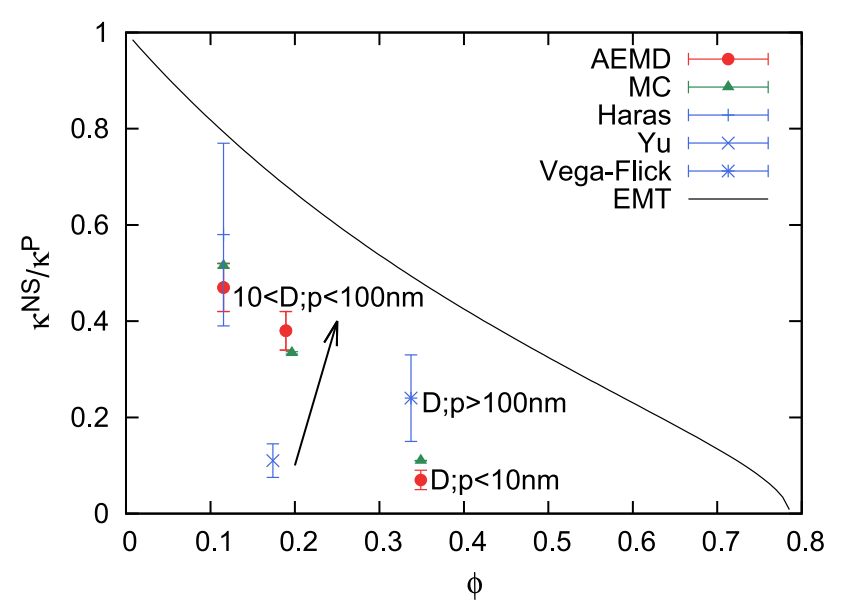

Fig. 5. Thermal conductivity reduction due to nanostructuration. The MD results are reported in red, the MC results in green, and the experimental values of Haras et al. [7], Yu et al. [5] and Vega-Flick et al. [8] in blue. The full line is the continuum result from the effective medium theory. (For interpretation of the references to colour in this figure legend, the reader is referred to the web version of this article.)

the phonons are populated according to the Bose-Einstein distribution in the MC calculations, but the MD trajectories are classical).

Finally, we have calculated the thermal conductivity following the effective medium theory (EMT). Within EMT, the heat equation is solved in a continuous and holey bulk as a function of the volume fraction, as presented in Appendix (dark line in Fig. 5). The result, that gives the macroscopic limit of thermal conductivity reduction by hole hollowing, is discussed in the next section.

\section{Discussion}

The thermal conductivity reduction, defined as the ratio of the thermal conductivity of the nanostructured membrane $\kappa^{\text {ns }}$ over the thermal conductivity of the plain membrane, $\kappa^{\mathrm{ns}} / \kappa^{\mathrm{P}}$ is plotted in Fig. 5 as a function of the volume fraction $\phi$, defined as the ratio of hole volume over total volume. The experimental and theoretical results are distributed on 3 scales: (a) pitch and diameters smaller than $10 \mathrm{~nm}(D ; p<10 \mathrm{~nm})$, (b) in the deca-nanometer range $(10<D ; p<100 \mathrm{~nm})$ and (c) greater than $100 \mathrm{~nm}$ $(D ; p>100 \mathrm{~nm})$. In the range (a), not achievable in practice even by the most advanced technological recipes, we find the MD and MC calculations with the small pattern [11]. In the range (b), we find the experimental and theoretical calculations on the pattern $\mathrm{H}$ and $\mathrm{Y}$, and in the range (c) larger patterns as fabricated by Vega-Flick et al. [8], and out of reach of the MD calculations. All the results lie below the macroscopic limit given by the effective medium theory, as expected from the downscaling of the membranes dimensions. MD and MC results are in good agreement, as already mentioned in Section 3. At fix volume fraction, the thermal conductivity reduction is more pronounced when the scaling goes down, as evidenced for $\phi \approx 0.35$ where we can compare the EMT result, the measurement by Vega-Flick (scale $D ; p>100 \mathrm{~nm}$ ) and the MD and MC calculation on the small pattern $(D ; p<10 \mathrm{~nm})$. Concerning the systems in the deca-nanometric range $(10<D ; p<100 \mathrm{~nm})$, they have comparable volume fraction $(0.1<\phi<0.2)$ and the thermal conductivity reduction is of the same order (0.4-0.5) at the exception of the measurement of Ref. [5] (0.1).

In order to investigate the origin of this difference, we have carried out additional MC calculations using a specularity parameter $p$ that corresponds to a full diffuse scattering at the top and bottom surfaces. The thermal conductivities of the plain and nanostructured membranes are reduced respectively to 22.2 and $7.4 \mathrm{~W} \mathrm{~K}^{-1}$ $\mathrm{m}^{-1}$. The reduction factor is the same, $\approx 0.33$. However, in the hypothesis that the surface roughness has been increased during the step of hole hollowing, the thermal conductivity would then decrease from $\approx 45 \mathrm{~W} \mathrm{~K}^{-1} \mathrm{~m}^{-1}$ (plain membrane with smooth surfaces) to $\approx 7 \mathrm{~W} \mathrm{~K}^{-1} \mathrm{~m}^{-1}$ (nanostructured membrane with rough surfaces). The corresponding reduction factor (0.15) would then be in agreement with the measurements.

\section{Conclusion}

In conclusion, we have exploited the approach-to-equilibrium methodology to calculate the thermal conductivity of real nanostructures having dimensions in the deca-nanometric range. We have shown that for volume fractions around 0.1-0.2, the thermal conductivity reduces by a factor of 2 approximately compared to the plain membrane. This reduction level is in agreement with some of the reported measurements in the literature, but can not explain the strong reduction obtained in other works. We have used Monte Carlo simulations to rationalize a stronger reduction in the case where the hole fabrication would increase the surface roughness. This assumption should of course be validated experimental and non-trivial nano-characterisation of the surface state. Moreover in this case, the low thermal conductivity would be adapted to an efficient thermoelectric module, but the electronic conductivity could be impacted by the high surface roughness. In order to further decrease the thermal conductivity of crystalline Si membranes with a reduced surface roughness, a larger volume fraction of holes or a stronger downscaling are two possible routes.

\section{Conflicts of interest}

There are no conflicts of interest to declare.

\section{Acknowledgements}

Calculations were performed on the facilities of IEMN (clustphy), of IJL-LEMTA (Ermione cluster) and on the computational ressources from GENCI (Grand Equipement National de Calcul Intensif) (Grants No. [2014]-x2014097186 and [2016:2017]A001097600).

\section{Appendix A. Thermal conductivity from the effective medium theory}

We consider a heterogeneous medium characterized by a position-dependent thermal conductivity $\kappa$. In a steady state the Fourier heat equation reads:

$\vec{\nabla} \kappa \cdot \vec{\nabla} T+\kappa \nabla^{2} T=0$.

We assume that $\kappa$ depends on $y$ and $z$, being however constant along the $x$ direction, i.e. $\kappa=\kappa(y, z)$. Moreover, $\kappa(y, z)$ is a doubly periodic function with periods $L_{y}$ and $L_{z}$ in the $y$ and $z$ directions, respectively (rectangular symmetry). The length $L$ of the overall system in the $z$ direction is much larger than $L_{z}$. Further, the system is assumed to be infinite along the other directions $x$ and $y$. This system is subjected to the boundary conditions $T(0, y)=T_{1}$ and $T(L, y)=T_{2}$, which are able to mimic a remotely applied heat flux. In order to solve Eq. (A.1) in a region far from the boundaries, we define the Fourier transforms of the conductivity $\kappa$ and the scalar field $T$ :

$\kappa(y, z)=\sum_{n=-\infty}^{\infty} \sum_{m=-\infty}^{\infty} \kappa_{n, m} e^{2 \pi i\left(\frac{n y}{L_{y}}+\frac{m z}{L_{z}}\right)}$,
$T(y, z)=\frac{\left(T_{2}-T_{1}\right) z}{L}+\sum_{n=-\infty}^{\infty} \sum_{m=-\infty}^{\infty} T_{n, m} e^{2 \pi i\left(\frac{n y}{L_{y}}+\frac{m z}{L_{z}}\right)}$. 


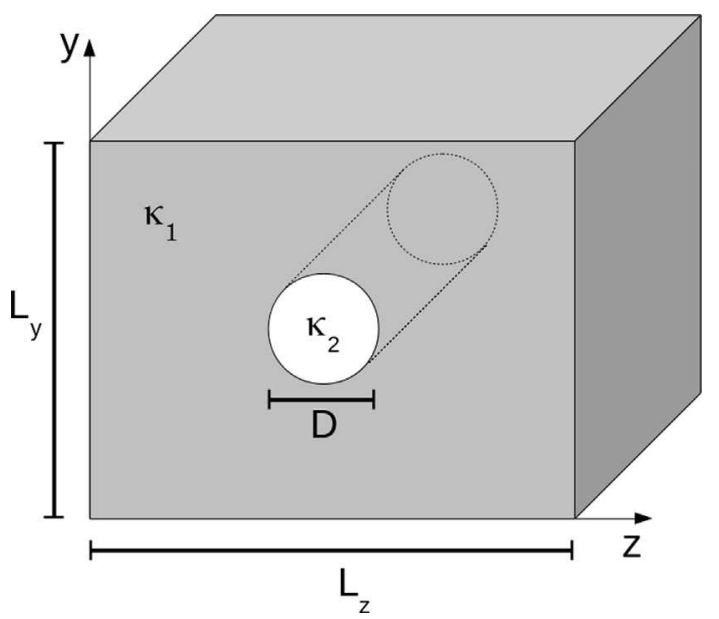

Fig. A.6. Cylindrical inclusion of thermal conductivity $\kappa_{2}$ in a medium of thermal conductivity $\kappa_{1}$.

In terms of Fourier components, Eq. (A.1) reads

$\sum_{n=-\infty}^{\infty} \sum_{m=-\infty}^{\infty} T_{n, m} \kappa_{k-n, h-m} 2 \pi\left(\frac{n k}{L_{y}^{2}}+\frac{m h}{L_{z}^{2}}\right)-\kappa_{k, h} i \frac{h}{L_{z}} \frac{\left(T_{2}-T_{1}\right)}{L}=0$,

which is a set of infinite equations $(-\infty<k, h<+\infty)$ that must be numerically solved to get the unknowns $T_{n, m}$. Here, we have

$\kappa_{k, h}=\frac{1}{L_{y} L_{z}} \int_{0}^{L_{y}} \int_{0}^{L_{z}} \kappa(y, z) e^{-2 \pi i\left(\frac{k y}{L_{y}}+\frac{h z}{L_{z}}\right)} d y d z$

The effective conductivity $\kappa_{\text {eff }}$ along the $z$ direction is defined by the ratio of the average heat flux $\left\langle J_{z}\right\rangle=\left\langle-\kappa \frac{\partial T}{\partial z}\right\rangle$ to the average thermal gradient $\left\langle-\frac{\partial T}{\partial z}\right\rangle=-\frac{\left(T_{2}-T_{1}\right)}{L}$. After some straightforward calculation, we get

$\kappa_{\text {eff }}=\kappa_{0,0}-\sum_{n=-\infty}^{\infty} \sum_{m=-\infty}^{\infty} T_{n, m} \frac{2 \pi i m}{L_{z}} \kappa_{-n,-m}$.

If we take into consideration cylindrical inclusions of diameter $D$, placed at the center of each cell, we are able to homogenize a doubly periodic array of cylindrical inhomogeneities. The cylinders have a thermal conductivity $\kappa_{2}$ and are embedded in a matrix of conductivity $\kappa_{1}$ (see Fig. A.6). Hence, we can calculate through Eq. (A.5) the Fourier coefficients of the thermal conductivity, as follows

$$
\begin{aligned}
& \kappa_{0,0}=\kappa_{1}(1-f)+\kappa_{2} f \\
& \kappa_{n, m}=\left(\kappa_{2}-\kappa_{1}\right) \frac{(-1)^{n+m} \sqrt{\phi}}{\sqrt{\pi\left(n^{2}+m^{2}\right)}} J_{1}\left(2 \sqrt{\phi \pi\left(n^{2}+m^{2}\right)}\right): \text { for }(n, m) \neq(0,0)
\end{aligned}
$$

where $J_{1}$ is the Bessel function of the first kind and order one and $\phi=\pi D^{2} /\left(4 L_{y} L_{z}\right)$ is the volume fraction of the embedded cylinders.

The effective thermal conductivity of the porous system addressed in this paper can be eventually calculated through Eq. (A.6), where the coefficients $T_{n, m}$ are numerically evaluated by means of Eqs. (A.4) and (A.7) with $L_{y}=L_{z}$ and $\kappa_{2}=0$.

\section{References}

[1] A.I. Hochbaum, R. Chen, R.D. Delgado, W. Liang, E.C. Garnett, M. Najarian, A. Majumdar, P. Yang, Nature 451 (2008) 163.

[2] J. Kim, J.-H. Bahk, J. Hwang, H. Kim, H. Park, W. Kim, Phys. Stat. Solidi RRL 7 (2013) 767-780.

[3] J. Lee, W. Lee, J. Lim, Y. Yu, Q. Kong, J.J. Urban, P. Yang, Nano Lett. 16 (2016) 4133-4140.

[4] J. Tang, H.T. Wang, D.Y. Lee, M. Fardy, Z. Huo, T.P. Russell, P. Yang, Nano Lett. 10 (2010) 4279-4283.

[5] J.-K. Yu, S. Mitrovic, D. Tham, J. Varghese, J.R. Heath, Nat. Nanotech. 5 (2010) $718-721$.

[6] M. Nomura, J. Nakagawa, Y. Kage, J. Maire, D. Moser, O. Paul, Appl. Phys. Lett. 106 (2015) 143102

[7] M. Haras, V. Lacatena, T. Moussa Bah, S. Didenko, J.-F. Robillard, S. Monfray, T. Skotnicki, E. Dubois, IEEE Electron Dev. Lett. 37 (2016) 1358-1361.

[8] A. Vega-Flick, R.A. Duncan, J.K. Eliason, J. Cuffe, J.A. Johnson, J.-P.M. Peraud, L. Zeng, Z. Lu, A.A. Maznev, E.N. Wang, J.J. Alvarado-Gil, M. Sledzinska, C.M. Sotomayor Torres, G. Chen, K. Nelson, AIP Adv. 6 (2016) 121903.

[9] A.S. Henry, G. Chen, J. Comput. Theo. Nanosci. 5 (2008) 141-152.

[10] K.T. Regner, D.P. Sellan, Z. Su, C.H. Amon, A.J.H. McGaughey, J.A. Malen, Nat. Comm. 4 (2013), 1640:1:7.

[11] V. Lacatena, M. Haras, J.-F. Robillard, S. Monfray, T. Skotnicki, E. Dubois, Appl. Phys. Lett. 106 (2015) 114104.

[12] R. Zwanzig, Ann. Rev. Phys. Chem. 16 (1965) 67-102.

[13] J. Tersoff, Phys. Rev. B 38 (1988) 9902.

[14] I.T. Todorov, W. Smith, K. Trachenko, M.T. Dove, J. Mater. Chem. 16 (2006) 1911. http:://www.ccp5.ac.uk/DL_POLY/ .

[15] E. Lampin, Q.H. Nguyen, P.-A. Francioso, F. Cleri, Appl. Phys. Lett. 100 (2012) 131906.

[16] E. Lampin, P.L. Palla, P.-A. Francioso, F. Cleri, J. Appl. Phys. 114 (2013) 033525

[17] S. Nosé, A molecular dynamics method for simulations in the canonical ensemble, Mol. Phys. 52 (1984) 255-268.

[18] W.G. Hoover, Canonical dynamics: equilibrium phase-space distributions, Phys. Rev. A 31 (1985) 1695-1697.

[19] H. Zaoui, P.L. Palla, F. Cleri, E. Lampin, Phys. Rev. B 94 (2016) 054304.

[20] H. Zaoui, P.L. Palla, F. Cleri, E. Lampin, Phys. Rev. B 95 (2017) 104309.

[21] E. Chávez-Ángel, J.S. Reparaz, J. Gomis-Bresco, M.R. Wagner, J. Cuffe, B. Graczykowski, A. Shchepetov, H. Jiang, M. Prunnila, J. Ahopelto, F. Alzina, C.M. Sotomayor, Torres APL Mater. 2 (2014) 012113.

[22] S. Neogi, J.S. Reparaz, L.F.C. Pereira, B. Graczykowski, M.R. Wagner, M Sledzinska, A. Shchepetov, M. Prunnila, J. Ahopelto, C.M. Sotomayor-Torres, D. Donadio, ACS Nano 9 (2015) 3820-3828.

[23] V. Jean, S. Fumeron, K. Termentzidis, S. Tutashkonko, D. Lacroix, J. Appl. Phys. 115 (2014) 024304.

[24] V. Jean, S. Fumeron, K. Termentzidis, X. Zianni, D. Lacroix, Int. J. Heat Mass Trans. 86 (2015) 648-655.

[25] M. Verdier, R. Anufriev, A. Ramiere, K. Termentzidis, D. Lacroix, Phys. Rev. B 95 (2017) 205438

[26] M.G. Holland, Phys. Rev. 134 (1964) A471-A480.

[27] E. Pop, R.W. Dutton, K.E. Goodson, J. Appl. Phys. 96 (2004) 4998.

[28] M.Z. Bazant, E. Kaxiras Phys. Rev. Lett., 1996, 77, 4370. M.Z. Bazant, E. Kaxiras, J.F. Justo Phys. Rev. B, 1997, 56, 8542. J.F. Justo, M.Z. Bazant, E. Kaxiras, V.V. Bulatov, S. Yip Phys. Rev. B, 1998, 58, 2539 